\title{
Angular analyses of $b \rightarrow s \ell \ell$ modes at LHCb
}

\author{
Eluned Smith* \\ RWTH, Aachen \\ E-mail: eluned.a.smith@cern.ch
}

The study of rare decays mediated via $b \rightarrow s \ell \ell$ transitions allows for new physics searches which are sensitive to mass scales up to $\mathscr{O}(100) \mathrm{TeV}$. Sensitivity to new physics can be increased via angular analyses, as angles involve ratios of observables, causing angular coefficients to have reduced theoretical uncertainties. There continue to be significant tensions with the Standard Model across a range of experimental observables associated with $b \rightarrow s \ell l$ decays, including in angular observables. These proceedings outline the latest analyses involving this class of decays at $\mathrm{LHCb}$, with a focus on angular analyses and modes involving muons.

The International Conference on B-Physics at Frontier Machines - BEAUTY2018

6-11 May, 2018

La Biodola, Elba Island, Italy

* Speaker.

${ }^{\dagger}$ On behalf of the LHCb collaboration 


\section{Rare decays and angular analyses}

The majority of the decays discussed in these proceedings occurs via $b \rightarrow s \ell \ell$ transitions, mediated with Flavour-Changing Neutral Currents (FCNC), as indicated schematically in figure 1.

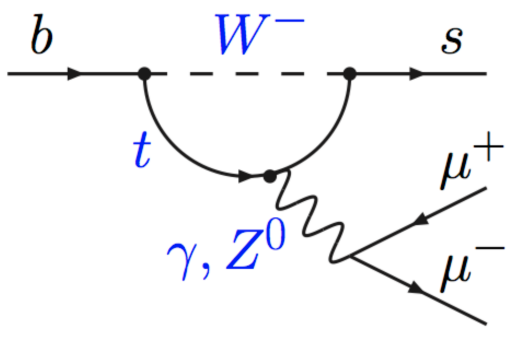

Figure 1: Example Feynman diagram for a $b \rightarrow s \ell \ell$ transition

As FCNCs cannot occur at tree-level, $b \rightarrow$ sl $\ell$ decays are suppressed in the Standard Model (SM). This suppression makes the experimental observables associated with these decays more sensitive to new physics (NP) effects. Furthermore, the inherent higher-order nature of these decays renders them sensitive to diagrams mediated via off-shell NP particles and, as such, allows for the testing of NP models involving mass scales of up to $\mathscr{O}(100) \mathrm{TeV}$ [1].

In order to facilitate the search for signs of NP, measured experimental observables must be compared with their values as predicted by the SM. These SM predictions are performed within the context of effective field theory, exploiting the different mass scales involved in $b \rightarrow s \ell \ell$ transitions, which allows one to integrate out the heavier degrees of freedom and represent them with constants referred to as Wilson coefficients.

As such, experimental observables can be described in terms of combinations of Wilson coefficients, and other nuisance parameters such as form factors, which describe the purely hadronic parts of the process. Due to the heavier nature of the physics that they describe, Wilson coefficients have small theoretical uncertainties when compared with the uncertainties associated with the form factors describing softer QCD effects. The theoretical uncertainty on angular coefficients is reduced as these observables are dependent on ratios of decay properties, in which some theoretical uncertainties cancel. In these proceedings the status and results from various rare decay analyses at LHCb are discussed, with a focus on angular analyses involving muons.

\section{Angular analysis of the decay $B^{0} \rightarrow K^{* 0} \mu^{+} \mu^{-}$}

This section focuses on the analysis performed by the LHCb collaboration in Ref. [2], using the $3 \mathrm{fb}^{-1}$ of data collected during the Run 1 data taking of the LHC.

The CP-averaged angular decay rate of the decay $B^{0} \rightarrow K^{* 0} \mu^{+} \mu^{-}$, where the $K^{* 0}$ decays as $K^{* 0} \rightarrow K^{+} \pi^{-}$, can be expressed in terms of bins averaged over the square of the dimuon invariant mass $\left(q^{2}\right)$ as

$$
\frac{1}{d(\Gamma+\bar{\Gamma}) / d q^{2}} \frac{d^{3}(\Gamma+\bar{\Gamma})}{d \vec{\Omega}}=\sum_{i=1} I_{i} \mathrm{f}_{i}(\Omega),
$$


where $\vec{\Omega}=\mathrm{d} \cos \left(\theta_{K}\right), \mathrm{d} \cos \left(\theta_{l}\right), \phi$ and the angles $\theta_{k, l}, \phi$ are defined schematically in figure 2 .

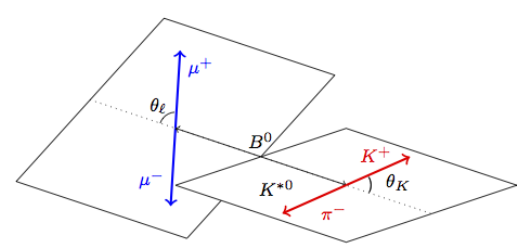

(a) $\theta_{K}$ and $\theta_{\ell}$ definitions for the $B^{0}$ decay

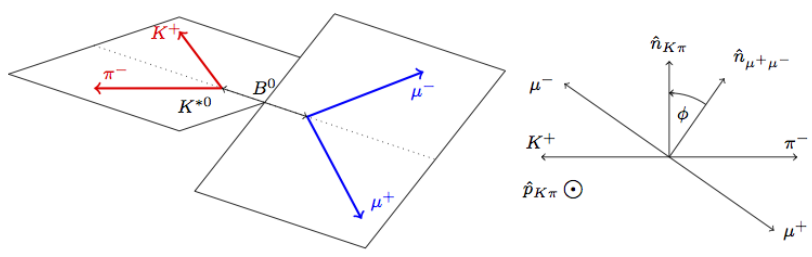

(b) $\phi$ definition for the $B^{0}$ decay

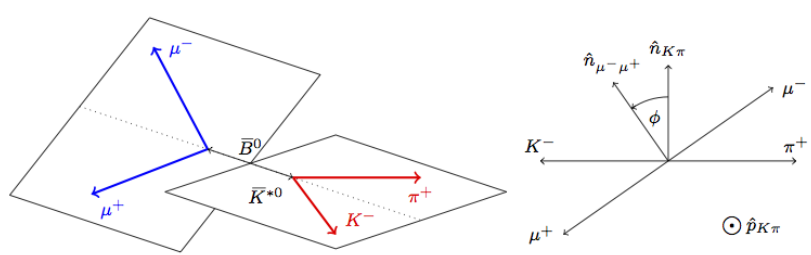

(c) $\phi$ definition for the $\bar{B}^{0}$ decay

Figure 2: Definition for the angles used in the $B^{0} \rightarrow K^{* 0} \mu^{+} \mu^{-}$angular analysis.

The constants $I_{i}$ are either CP-even $\left(S_{j}\right)$ or CP-odd $\left(A_{k}\right)$ angular coefficients, which are particularly sensitive to the Wilson coefficients $C_{9,10}$. An additional suffix s or c is conventionally added to some of the $I_{i}$ terms to indicate that they have a $\sin ^{2}\left(\theta_{k}\right)$ or $\cos ^{2}\left(\theta_{k}\right)$ dependence.

To extract the angular coefficients, the expression in equation 2.1 is fitted to the angles, $\vec{\Omega}$, in data, in bins of $q^{2}$, with the $m_{K \pi \mu \mu}$ mass distribution being used to distinguish between background and signal. In turn, the $m_{K \pi}$ distribution is used to distinguish between the resonant signal coming from the $K^{* 0}$ vector resonance and the non-resonant $K \pi$ spin- 0 component (whereby the presence of the latter adds an additional 7 angular coefficients to the decay rate, which are treated as nuisance parameters).

The charmonium mediated tree-level decays, $B^{0} \rightarrow J / \psi\left(\rightarrow \mu^{+} \mu^{-}\right) K^{* 0}$ and $B^{0} \rightarrow \psi(2 S)(\rightarrow$ $\left.\mu^{+} \mu^{-}\right) K^{* 0}$, have the same final state as $B^{0} \rightarrow K^{* 0} \mu^{+} \mu^{-}$and a much higher decay rate, and as such, vetoes must be used to remove contributions from these decays. This is achieved by vetoing events in the $q^{2}$ spectrum which lie near the $J / \psi$ and $\psi(2 S)$ masses, as demonstrated in figure 3, which shows the invariant mass, $m_{K \pi \mu \mu}$, against $q^{2}$ for all data events collected in Run 1 by the LHCb experiment and passing the relevant selection criteria.

The similarity of the charmonium mediated decays to the signal channel is exploited however, by using these decays as control channels, helping to reduce systematic uncertainties on results. 


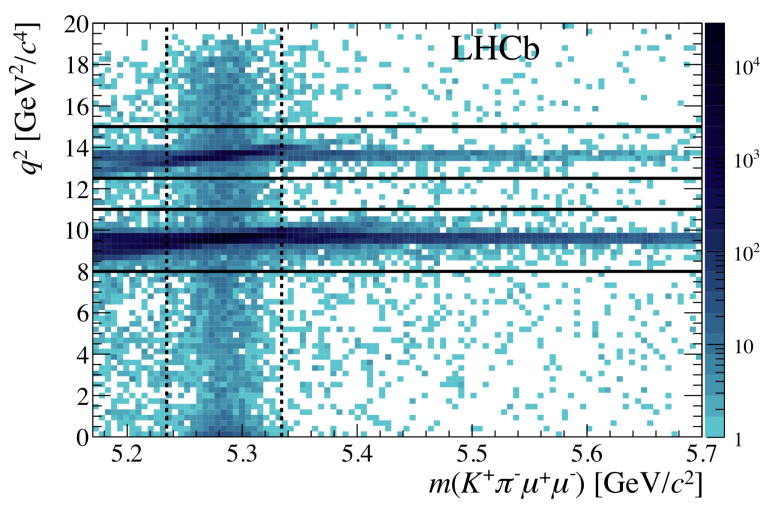

Figure 3: The invariant mass, $m_{K \pi \mu \mu}$, against $q^{2}$ for all $B^{0} \rightarrow K^{* 0} \mu^{+} \mu^{-}$signal candidates collected in Run 1 by the $\mathrm{LHCb}$ experiment and passing the selection criteria. The decay $B^{0} \rightarrow K^{* 0} \mu^{+} \mu^{-}$is clearly visible inside the dashed vertical lines. The horizontal lines denote the charmonium regions, where the tree-level decays $B^{0} \rightarrow J / \psi\left(\rightarrow \mu^{+} \mu^{-}\right) K^{* 0}$ and $B^{0} \rightarrow \psi(2 S)\left(\rightarrow \mu^{+} \mu^{-}\right) K^{* 0}$ dominate. These candidates are excluded from the analysis.

The fit to the angles $\vec{\Omega}$ for Run1 LHCb data in the region $1.1<q^{2}<6.0 \mathrm{GeV} / c^{2}$ is shown in figure 4 and the resulting values for some of the angular coefficients are shown in figure 5. The angular coefficients shown are $F_{L}\left(=S_{1 c}\right)$ and $A_{F B}\left(\propto S_{6 s}\right)$. Both angular coefficients shown in figure 5, along with most other angular coefficients, are in good agreement with the SM.
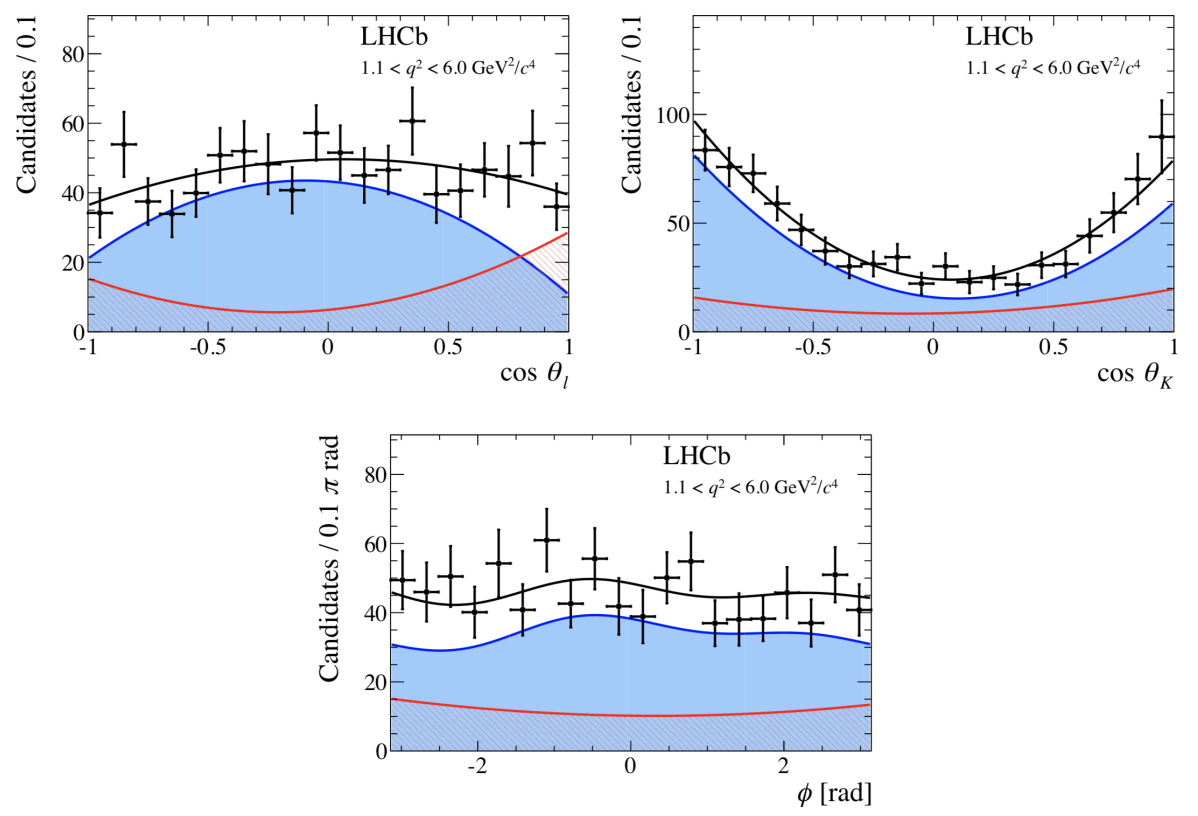

Figure 4: Fit of LHCb Run 1 data to angles in the $q^{2}$ range $1.1<q^{2}<6.0 \mathrm{GeV} / c^{2}$. Blue represents the projection of the signal PDF and red the projection of the background PDF.

The theoretical error on the angular coefficients can be further reduced by taking combinations of the $I_{i}$ terms such that the form factor uncertainties cancel at first order. These are referred to as $P_{i}^{\prime}$ variables [3]. There is a significant discrepancy between the SM predictions and the 

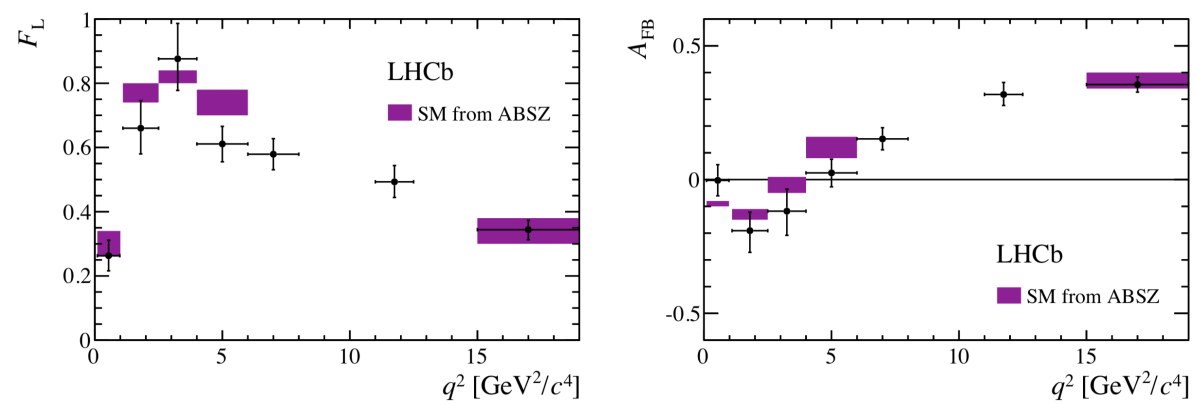

Figure 5: Results of two of the angular coefficients extracted from the angular fits performed in bins of $q^{2}$. See text for more explanation.

measured values for the variable $P_{5}^{\prime}$. This discrepancy is observed across a range of experiments, as demonstrated in figure 6, where results are shown from the collaborations: LHCb[2], OMS [4], Belle[5] and ATLAS[6]. This tension amounts to a 3.4 $\sigma$ deviation from the SM when considering the $\mathrm{LHCb}$ data alone and assuming that the discrepancy comes from a shift in $C_{9}$ alone. There is also some tension seen at low $q^{2}$ in the $B^{0} \rightarrow K^{* 0} \mu^{+} \mu^{-}$branching fraction measurement, where the observed branching fraction is below what is expected in the SM, with similar trends in other $b \rightarrow$ sll transitions being described further in the following section.

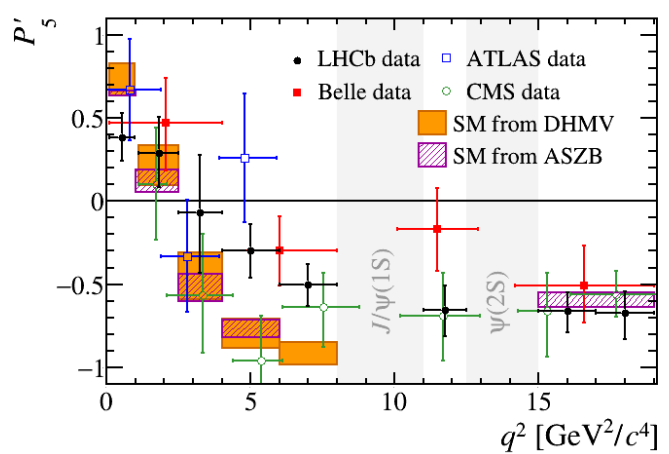

Figure 6: The measured value of the angular coefficient $P_{5}^{\prime}$ by different experiments compared against different theoretical predictions.

\section{The decay $B_{s}^{0} \rightarrow \phi \mu^{+} \mu^{-}$}

As with the decay $B^{0} \rightarrow K^{* 0} \mu^{+} \mu^{-}$, the decay $B_{s}^{0} \rightarrow \phi \mu^{+} \mu^{-}$is a $b \rightarrow$ sl $\ell$ FCNC, whose angular coefficients and decay rate can be used as tests of the SM. A similar angular analysis as described for $B^{0} \rightarrow K^{* 0} \mu^{+} \mu^{-}$decays was also performed by the LHCb collaboration on Run 1 data in Ref. [7] using $B_{s}^{0} \rightarrow \phi \mu^{+} \mu^{-}$decays. A key difference here however is that, as the $\phi$ decays to two kaons, the $B_{s}^{0}$ cannot be distinguished from the $\bar{B}_{s}^{0}$ decay without flavour tagging, where the introduction of flavour tagging would result in a large lose of statistics, and therefore the $P_{5}^{\prime}$ variable can not be accessed. 
In general, good agreement is seen for this mode between the SM predictions and the angular coefficients. The branching fraction however shows significant deviations from the SM, as shown in figure 7, top left. Again, here the branching fraction is measured relative to the charmonium mode $B_{s}^{0} \rightarrow J / \psi \phi$, which helps to reduce systematic uncertainties.

The trend seen in the $B_{s}^{0} \rightarrow \phi \mu^{+} \mu^{-}$branching fraction at low $q^{2}$ is mirrored elsewhere, as further highlighted in figure 7 (bottom and top right), where the measured branching fractions for various other $b \rightarrow s \mu \mu$ processes [8] are shown plotted against their SM predictions.
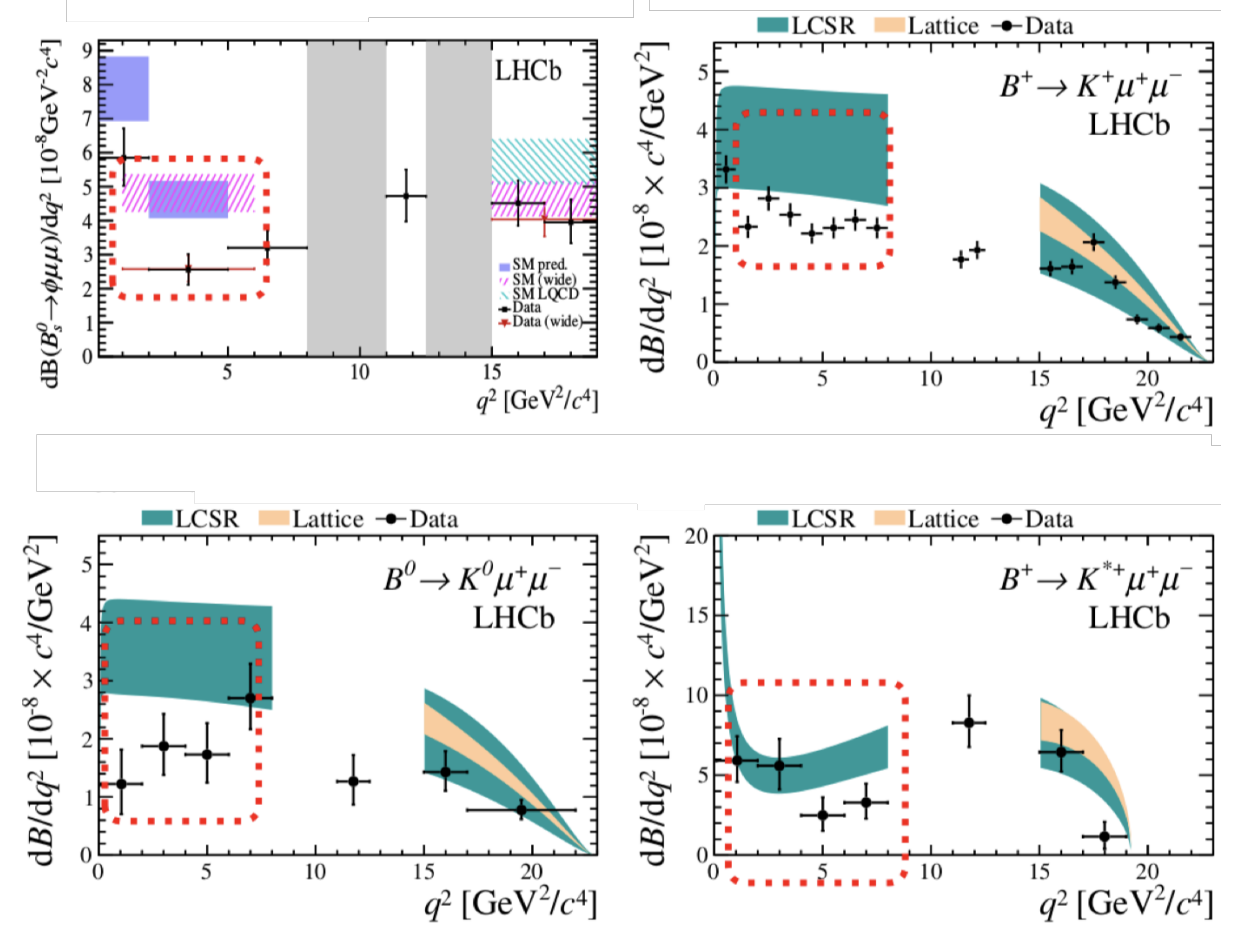

Figure 7: Differential branching fraction for $b \rightarrow s \mu \mu$ transitions with different spectator quarks, as indicated on the plots, against SM predictions. Red boxes highlight persistent tension with SM predictions at low $q^{2}$.

\section{Global fits to $b \rightarrow s \ell \ell$ results and measuring charm-loop interference}

There have been a number of measurements involving $b \rightarrow s \ell \ell$ transitions which show tension with the SM. These include the above-mentioned angular analyses, but also measurements involving the ratios $R_{X}=\frac{\mathscr{B}\left(b \rightarrow X \mu^{+} \mu^{-}\right)}{\mathscr{B}\left(b \rightarrow X e^{+} e^{-}\right)}$, which should be near-unity in the SM [9], and are theoretically very clean. Interestingly, the values of $R_{X}$ measured indicate a deficit of muons [10,11], which is compatible with the tensions observed in the branching fraction and angular analyses of $b \rightarrow s \mu \mu$ transitions involving muons.

To quantify these observed tensions across different data sets further, one can perform a global fit across different experimental results. In these global fits the form factors and nuisance parameters are constrained and the Wilson coefficients are fitted for. 
An example of such a fit is shown in figure 8, taken from Ref. [12], with similar fits carried out across a range of studies by different authors $[13,14]$

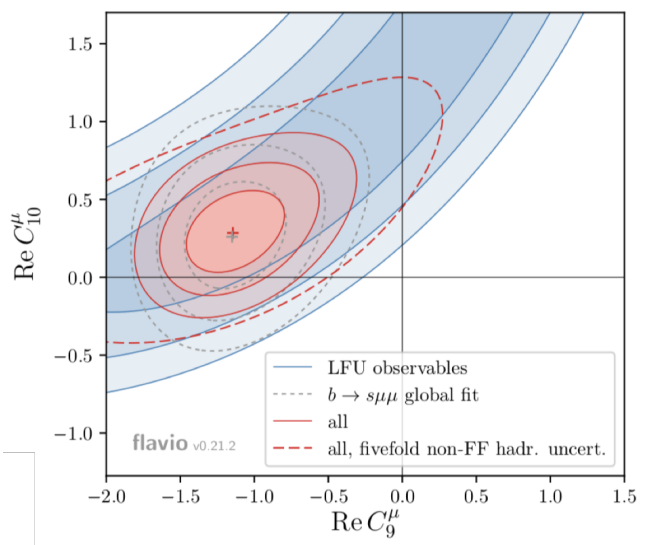

Figure 8: Results of the best value of the Wilson coefficients from the global fit taken from Ref. [12], assuming all other coefficients are SM-like. LFU in legend indicates the results when only considering the Lepton Flavour Universality variables $R_{X}$.

Depending on which Wilson coefficients are allowed to float, deviations of up to $4 \sigma$ from the SM are observed when only the theoretically clean $R_{X}$ observables are taken into account. These tensions go up to above $5 \sigma$ when results with larger theoretical uncertainties, such as those from angular analyses, are taken into account. In the case that new physics is observed, such angular observables would be vital for understanding the size and handedness of the NP contribution.

It is also possible however, that the deficit in muons at low $q^{2}$ with respect to the SM could be caused by incorrect parametrisation of the destructive interference from long distance effects from the $c \bar{c}$ vector resonances [15]. This interference would affect the Wilson coefficient $C_{9}$, as this coefficient represents the vector contribution to the integrated-out $b \rightarrow s \ell \ell$ loop. In order to investigate this further, one can use the data itself to parameterise this possible interference effect. This can be achieved by fitting across all $q^{2}$ and fitting directly for the Wilson coefficients, whilst modelling $C_{9}$ as the true value of $C_{9}$ plus an additional contribution from charmonium vector resonances, $\mathrm{Y}\left(q^{2}\right)$ :

$$
C_{9}^{e f f}=C_{9}+Y\left(q^{2}\right)
$$

Here, $\mathrm{Y}\left(q^{2}\right)$ is expressed as a sum of all possible charmonium resonances up to the open charm threshold, where each resonance is described by a line-shape $A_{\text {res }}$, generally a Breit-Wigner, a magnitude $\left(\eta_{j}\right)$ and its phase $\left(\delta_{j}\right)$ relative to $C_{9}$. This gives:

$$
C_{9}^{e f f}=C_{9}+\sum_{i} \eta_{i} \delta_{i} A_{r e s, i}
$$

This fit model was applied to $B^{+} \rightarrow K^{+} \mu^{+} \mu^{-}$Run 1 data from LHCb in Ref. [16]. Fitting the decay rate, expressed in terms of $C_{9}^{e f f}, C_{10}$, form factors and other nuisance parameters, the magnitudes and phases were found to be small and, within the given limitations of the model, not large enough to explain the large discrepancies seen in $C_{9}$. The interference between the long and short distance components of the fit is demonstrated in figure 9 . 


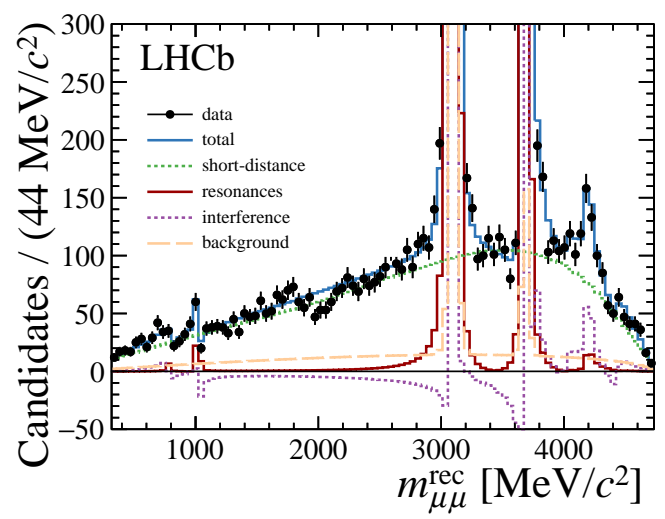

Figure 9: One of the unbinned fits to $B^{+} \rightarrow K^{+} \mu^{+} \mu^{-}$data. The interference between the long-distance and short-distance physics is indicated on the figure.

\section{5. $b \rightarrow d \ell \ell$ transitions}

The increasing statistics being collected by the LHCb collaboration means that measurements of decays with smaller branching fractions, or from mothers with a smaller fragmentation fraction such as $\Lambda_{b}^{0}$,s, are now becoming possible. These includes measurements of $b \rightarrow$ sll transitions in baryons as well as Cabibbo suppressed $b \rightarrow d \ell \ell$ transitions.

The measurement of $b \rightarrow d \ell \ell$ transitions is of interest, not just because they can be used to perform similar tests of the SM as per with the $b \rightarrow s \ell \ell$ decays, but also because the combination of $b \rightarrow d \ell \ell$ transitions with their $b \rightarrow s \ell \ell$ equivalent allows for a test of Minimal Flavour Violation.

First evidence of the $b \rightarrow d \ell \ell$ mediated decay $B_{s}^{0} \rightarrow \bar{K}^{* 0} \mu^{+} \mu^{-}$was reported by LHCb in Ref. [17] and the mass fit can be seen in figure 10. The first observation of a $b \rightarrow d \ell \ell$ transition in

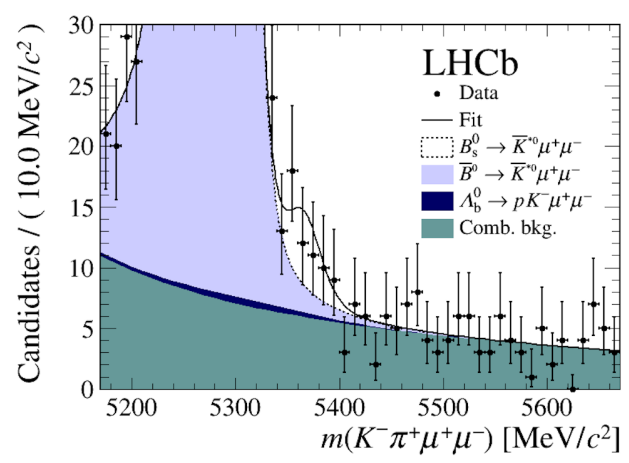

Figure 10: Fit to the invariant mass spectrum for $K \pi \mu \mu$ candidates. The decay $B_{s}^{0} \rightarrow \bar{K}^{* 0} \mu^{+} \mu^{-}$can be seen to the right of the larger $B^{0}$ peak.

the baryon sector was also reported by LHCb in Ref. [18].

\section{Conclusions}

The study of rare decays allows for tests of the Standard Model of up to heavier mass scales than those permitted by direct searches. There is currently much interest being generated in the 
flavour sector by observed deviations to the SM in experimental observables associated with $b \rightarrow s \ell \ell$ decays, including discrepancies in angular variables. Angular observables are of great interest as they help give more information of the nature of possible new physics models and a have a relatively low theoretical uncertainty when compared with observables such as decay rates. With the advent of both Run 3 at the LHC and the upgraded Belle 2 experiment, the flavour sector and rare decays will continue to be an interesting place to look for new physics.

\section{References}

[1] A. Burps, Posh FIN'S (2015) 003 doi:10.22323/1.220.0003 [arXiv:1505.00618 [hep-ph]].

[2] R. Haj at A. [LHCb Collaboration], JHEP 1602 (2016) 104 doi:10.1007/JHEP02(2016)104 [arXiv:1512.04442 [hep-ex]].

[3] S. Descotes-Genon, T. Hurth, J. Matias and J. Virto, JHEP 1305 (2013) 137 doi:10.1007/JHEP05(2013)137 [arXiv:1303.5794 [hep-ph]].

[4] A. M. Sirunyan Ut al. [CF'S Collaboration], Phys. Lett. B 781 (2018) 517 doi:10.1016/j.physletb.2018.04.030 [arXiv:1710.02846 [hep-ex]].

[5] S. Wehle et al. [Belle Collaboration], Phys. Rev. Lett. 118 (2017) no.11, 111801 doi:10.1103/PhysRevLett.118.111801 [arXiv:1612.05014 [hep-ex]].

[6] ATLAS conference note, ATLAS-CONF-2017-023

[7] R. Aaij et al. [LHCb Collaboration], JHEP 1509 (2015) 179 doi:10.1007/JHEP09(2015)179 [arXiv:1506.08777 [hep-ex]].

[8] R. Aaij at al. [LHCb Collaboration], JHEP 1406 (2014) 133 doi:10.1007/JHEP06(2014)133 [arXiv:1403.8044 [hep-ex]].

[9] G. Miller and F. Kruger, Phys. Rev. D 69 (2004) 074020 doi:10.1103/PhysRevD.69.074020 [hep-ph/0310219].

[10] R. Aaij et al. [LHCb Collaboration], JHEP 1708 (2017) 055 doi:10.1007/JHEP08(2017)055 [arXiv:1705.05802 [hep-ex]].

[11] R. Aaij et al. [LHCb Collaboration], Phys. Rev. Lett. 113 (2014) 151601 doi:10.1103/PhysRevLett.113.151601 [arXiv:1406.6482 [hep-ex]].

[12] W. Altmannshofer, P. Stangl and D. M. Straub, Phys. Rev. D 96 (2017) no.5, 055008 doi:10.1103/PhysRevD.96.055008 [arXiv:1704.05435 [hep-ph]].

[13] W. Altmannshofer, C. Niehoff, P. Stangl and D. M. Straub, Eur. Phys. J. C 77 (2017) no.6, 377 doi:10.1140/epjc/s10052-017-4952-0 [arXiv:1703.09189 [hep-ph]].

[14] T. Hurth, F. Mahmoudi and S. Neshatpour, Nucl. Phys. B 909 (2016) 737 doi:10.1016/j.nuclphysb.2016.05.022 [arXiv:1603.00865 [hep-ph]].

[15] S. Descotes-Genon, L. Hofer, J. Matias and J. Virto, JHEP 1412 (2014) 125 doi:10.1007/JHEP12(2014)125 [arXiv:1407.8526 [hep-ph]].

[16] R. Aaij et al. [LHCb Collaboration], Eur. Phys. J. C 77 (2017) no.3, 161 doi:10.1140/epjc/s10052-017-4703-2 [arXiv:1612.06764 [hep-ex]].

[17] R. Aaij et al. [LHCb Collaboration], arXiv:1804.07167 [hep-ex].

[18] R. Aaij et al. [LHCb Collaboration], JHEP 1704 (2017) 029 doi:10.1007/JHEP04(2017)029 [arXiv:1701.08705 [hep-ex]]. 